RESEARCH

\title{
RETINAL NEURODEGENERATION IN PATIENTS WITH MILD COGNITIVE IMPAIRMENT AND ALZHEIMER'S DISEASE AN OPTICAL COHERENCE TOMOGRAPHY STUDY
}

Turkish Journal of Geriatrics

DOI: 10.31086/tigeri.2021.214

2021; 24(2): 185-195

- Leyli Can Aynal ÖLÇÜcüOĞLU1 D

- Özlem Bizpınar MUNis ${ }^{1}$

- Huriye Hayat GÜVEN ${ }^{1}$

- Numan ERASLAN2

- Osman ÇELIKAY2.

- Selim Selçuk ÇOMOĞLU1

CORRESPONDANCE

${ }^{1}$ Leyli Can Aynal ÖLÇÜCÜOĞLU

University of Health Sciences, Dişkapı Yıldırım Beyazit Training and Research Hospital, Neurology, ANKARA, Turkey

\section{Phone: +905079157884 \\ e-mail: leylicanaynal@gmail.com}

Received: Feb 17, 2021

Accepted: Apr 16, 2021

1 University of Health Sciences, Dışkapı Yildırım Beyazıt Training and Research Hospital, Neurology, ANKARA, Turkey

2 University of Health Sciences, Dışkapı Yildırm Beyazıt Training and Research Hospital, Ophthalmology , ANKARA, Turkey

\section{Abstract}

Introduction: Recent developments suggest that optical coherence tomography can play an important role in diagnosing and following-up neurodegenerative diseases, including Alzheimer's disease. This study evaluates optical coherence tomography findings in mild cognitive impairment and middle-stage Alzheimer's disease patients.

Materials and Method: Seventy patients with amnestic type mild cognitive impairment, 54 patients with middle-stage Alzheimer's disease, and 54 cognitively healthy individuals were included. All study participants were evaluated with neuropsychological tests, ophthalmological examination, and optical coherence tomography. Ganglion cell complex and retinal nerve fiber layer thicknesses were measured, and comparisons were made between groups.

Results: Inferior temporal and inferior retinal nerve fiber layer thicknesses were thinner in the middle-stage Alzheimer's disease group than in the control and mild cognitive impairment groups. After statistical corrections, the inferior and inferior temporal retinal nerve fiber layers were thinner in the middlestage Alzheimer's disease group compared to the control and mild cognitive impairment groups. Besides, the nasal and nasal lower retinal nerve fiber layers were thicker than the control group. No statistically significant difference was found between the groups in terms of mean, superior and inferior ganglion cell complex thickness.

Conclusion: The results showed that retinal axon loss might develop in Alzheimer's disease. This loss may be focal, and the inferior temporal region may have selective sensitivity. Optical coherence tomography might reflect the neuropathological process in Alzheimer's disease, contribute to the diagnosis and follow-up. However, it is not helpful in the early diagnosis of Alzheimer's disease because of the lack of difference between mild cognitive impairment and control groups.

Keywords: Alzheimer Disease; Tomography, Optical Coherence; Retinal Degeneration; Dementia; Cognitive Dysfunction 


\section{INTRODUCTION}

Alzheimer's disease (AD) is a neurodegenerative disease characterized by the accumulation of neurofibrillary tangles containing intracellular hyperphosphorylated tau protein and extracellular amyloid $\beta(A \beta)$ protein in the brain, clinically resulting in dementia. It is accepted that these neuropathological changes begin 15-20 years before the clinical development of dementia. Mild cognitive impairment $(\mathrm{MCl})$ defines AD's prodromal stage, where objective cognitive problems but not functional loss are observed.

Advances in neuro-electrophysiological examinations and optical imaging have made it possible to evaluate neurodegenerative diseases' effects on the eye. Abnormal bioelectric activity and retinal microvascular changes, especially of retinal ganglion cells, photoreceptors, and the optic nerve, are associated with $A D$ and aging-related pathological processes and cognitive impairment $(1,2)$. The retina can offer an examination opportunity for early diagnosis of neurodegenerative diseases because it originates from the diencephalon during embryological development and contains a high density of neuronal cells and fibers. The possibility of the coexistence of retinal and brain pathologies is due to their common embryological origins and the similarity of blood-ocular and blood-brain barriers, common immunological responses, and similar sensitivity to damage (3). In the AD mouse model, amyloid- $\beta$ plaques, tau nodes, neurodegeneration, and astrogliosis have been detected in retinal ganglion cells starting from the pre-symptomatic stage (4). Postmortem studies also showed a decrease in the number of ganglion cells in the retina and thinning of the nerve fiber layer in $A D$ (5). In $A D$, it has been suggested that retinal nerve fiber layer (RNFL) thickness may decrease due to retrograde degeneration of retinal ganglion cell axons (6), and it has been shown that these changes in the retina may occur before the memory is affected (7).

With its high spatial resolution, optical coher- ence tomography (OCT) is an easily applicable and non-invasive examination that allows the detection of neuro-axonal degeneration by visualizing the retinal nerve tissue with biopsy-like sensitivity. OCT findings consistent with retinal neurodegeneration have been found in $A D$ patients compared to healthy controls, but few studies have evaluated OCT findings in $\mathrm{MCl}$ patients (8). On the other hand, it has been shown that RNFL thinness is associated with inferior cognitive functions in individuals without neurodegenerative diseases in baseline evaluation, and it has been reported that retinal neurodegeneration may be a preclinical biomarker for dementia $(9,10)$.

Determining the relationships between retinal changes and aging-related brain processes in healthy older adults, and distinguishing them from pathological changes associated with $A D$ also defining retinal markers, is essential for detecting the transition from the asymptomatic stage of $A D$ to the symptomatic pre-dementia stage or from the symptomatic pre-dementia stage to the dementia stage. OCT examination will be able to contribute to meeting this requirement in the following years. This study evaluates the OCT findings that may be markers of retinal neurodegeneration in middle-stage $A D$ and $\mathrm{MCl}$ patients and compares these findings with healthy individuals with normal cognitive functions.

\section{MATERIALS AND METHOD}

\section{Patient Selection}

This study included patients diagnosed with amnestic $\mathrm{MCl}(\mathrm{aMCl})$ and mid-stage $A D$, in addition to volunteers without cognitive impairment. The file records of the patients being followed up in the dementia outpatient clinic of the University of Health Sciences, Dışkapı Yıldırım Beyazıt Training and Research Hospital, Department of Neurology, were examined. The selected patients were re-evaluated with a neurological examination, neuropsychologi- 
cal tests, and brain imaging, and their diagnosis was confirmed. In addition, patients who applied to the neurology outpatient clinic with complaints of forgetfulness were evaluated similarly, and those diagnosed with aMCl and mid-stage $\mathrm{AD}$ were included in the study.

Neuropsychological tests (MMT, MOCA, clock scratching, geriatric depression scale) determined according to the patients' educational status included in the study and the volunteers included in the control group were performed. The patients were evaluated by two different neurology physicians and a psychologist, using neuropsychological tests and their addiction status in daily living activities and clinical observations. Amnestic $\mathrm{MCl}$ was diagnosed according to Peterson criteria, and mid-stage $A D$ diagnosis was made according to DSM-V and $\mathrm{Na}$ tional Institute on Aging-Alzheimer's Association (NIA-AA) criteria. Ophthalmologic examinations and OCT examinations, including corrected visual acuity, ocular motility, pupillary reflexes, slit-lamp biomicroscopy of the anterior segment, intraocular pressure measurement, and fundus examination, were performed in our hospital's ophthalmology department.

Exclusion criteria applied to patient and control groups included: failure to distinguish between $A D$ and other types of dementia clinically, mixed type dementia, non-amnestic $\mathrm{MCl}$, subjective cognitive impairment, cognitive impairment secondary to depression, systemic, psychiatric, or another neurological disease that may cause cognitive damage, and pathological findings that may affect cognitive functions in brain magnetic resonance imaging (MRI) (except atrophy), diabetes mellitus, inability to perform OCT due to cooperation difficulties, refractive error of more than 3 diopters spherical, 2 diopters cylindrical, glaucoma, retinal vascular occlusive disease, anterior ischemic optic neuropathy, ocular media opacity due to corneal abnormality, cataracts, macular edema, age-related macular degeneration interfering with ocular and/or OCT ex- amination, and retinal involvement due to diseases such as hypertension and diabetes mellitus.

\section{Optical Coherence Tomography}

After detailed ophthalmological examinations of the patients were performed, an OCT examination was performed by experienced practitioners on the same day using the RTVue SD-OCT system (RTVue-XR 100 Avanti software v.6.1, Optovue, Inc., Fremont, CA, USA). While evaluating the macula and optic nerve head, RNFL and ganglion cell complex (GCC) thickness were measured separately in both eyes. The measurements were repeated three times for each eye to reduce measurement errors.

The RSLT 3.45 protocol was used for peripapillary RNFL analysis, and peripapillary RNFL thickness was measured in a circular area of $3.45 \mathrm{~mm}$ diameters around the center of the optic disc. Peripapillary RNFL thickness maps were expressed as a numerical value, and each of the eight segments in four quadrants was evaluated by color-coding (superior: $46^{\circ}-135^{\circ}$; nasal: $316^{\circ}-45^{\circ}$ for right, $136^{\circ}-225^{\circ}$ for left; inferior: $226^{\circ}-315^{\circ}$; temporal: $136^{\circ}-225^{\circ}$ for right and $316^{\circ}-45^{\circ}$ for left). Parameter values within the normal range ( $p>5 \%)$ are in green, parameter values outside the normal range for age $(p<5 \%$ and $\geq 1 \%$ ) in yellow, and abnormal parameter values ( $p$ $<1 \%$, in red. The results were displayed on a color map with customized software with normative data adjusted for age and optical disc size.

RNFL thickness was obtained from all octameric section parameters: temporal upper (TU), superior temporal (ST), superior nasal (SN), nasal upper (NU), nasal lower (NL), inferior nasal (IN), inferior temporal (IT), and temporal lower (TL). Also, RNFL thicknesses for each of the temporal, superior, nasal, and inferior quadrants were obtained by averaging the octameric results: (TU TL) $\div 2$ for the temporal, (ST $\mathrm{SN}) \div 2$ for the superior, (NU NL) $\div 2$ for the nasal, and $(I T I N) \div 2$ for the inferior quadrants.

Macular GCC layer extends from the internal limiting membrane to the inner nuclear layer and 
includes the ganglion cell layer. GCC thickness was measured using the GCC protocol, which consisted of 15 vertical B scans (800 A scans for each) $7.0 \mathrm{~mm}$ long, each $0.50 \mathrm{~mm}$ separated, a single $7.0 \mathrm{~mm}$ long horizontal B scan (12.934 A scan). All measurements were centered on the macula. The center of the GCC scan was temporarily shifted $1.0 \mathrm{~mm}$ to better sample the temporal peripheral macula with the nasal visual field. Images were taken from a $6 \mathrm{~mm} \times 6$ $\mathrm{mm}$ area for GCC scanning.

Quantitative OCT examinations were carried out following APOSTEL recommendations (Advised Protocol for OCT Study Terminology and Elements) (11). For some patients and control groups, the OCT examination could only be performed in one eye, either due to difficulties in cooperation or the ocular reasons specified in the exclusion criteria. In these cases, data belonging to one eye were evaluated.

The study was conducted under the Helsinki Declaration and with the approval of the Ethics Committee of the University of Health Sciences, Dışkapı Yıldırım Beyazıt Training and Research Hospital (15.10.2018, 55/07). Written informed consent was obtained from all patients participating in the study.

\section{Statistical Analysis}

Data analysis was performed using IBM SPSS Statistics version 17.0 software (IBM Corporation, Armonk, NY, USA). The Kolmogorov-Smirnov test was used to determine whether the distributions of continuous variables were normal, and the Levene test was used to examine the assumption of homogeneity of variances. The descriptive statistics for continuous variables were expressed as median $(\mathrm{IQR})$; otherwise, the number of cases and percentages were used for categorical data. The continuous variables in which the parametrical test assumptions were not met were evaluated by the Kruskal-Wallis test. When the p-values from the Kruskal-Wallis test were statistically significant, Dunn-Bonferroni tests were used to determine which group differed from which others. Categorical data were analyzed by
Continuity corrected or Pearson's Chi-square tests, where applicable. The Wilcoxon Sign Rank test was used to evaluate whether the differences in the thickness of the ganglion cell complex and retinal nerve fiber layer between the left and right sides were statistically significant. Multiple linear regression analyses were performed to examine whether the difference in the thickness of the ganglion cell complex and retinal nerve fiber layer among groups persisted after adjusting for age and gender. Coefficients of regression, 95\% confidence intervals, and t-statistics for each independent variable were also calculated. Because of non-normal distribution, logarithmic transformation was used for the thickness of the ganglion cell complex and retinal nerve fiber layer in regression analysis. A p-value of less than 0.05 was considered statistically significant. However, for all possible multiple comparisons, the Bonferroni correction was applied for controlling Type I error.

\section{RESULTS}

Seventy patients diagnosed with $\mathrm{MCl}(28$ females, 42 males), 54 patients (34 females, 20 males) diagnosed with mid-stage $A D$, and 54 cognitively healthy individuals (32 females, 22 males) as the control group were included in the study. The groups showed a statistically significant difference in terms of mean age $(p<0.001)$. Patients in the $\mathrm{MCl}$ and middle-stage AD groups were found to be older than the control group $(p=0.048$ and $p<0.001$, respectively), while there was no statistically significant difference in age between the $\mathrm{MCl}$ and midstage AD groups $(p=0.188)$. There was a statistically significant difference between the groups in terms of gender distribution ( $p=0.021$ ). The proportion of males in the $\mathrm{MCl}$ group was higher than in the middle-stage $A D$ and control groups $(p=0.033$ and $p$ $=0.011$, respectively). On the other hand, there was no statistically significant difference between the middle-stage $A D$ and the control group in terms of female and male distribution ( $p=0.844$ ) (Table 1). 
It was found that MMT scores decreased in the control, $\mathrm{MCl}$, and mid-stage AD groups, respective$l y$, and there was a statistically significant difference between all three groups $(p<0.001)$ (Table 1).

The OCT evaluated 138 eyes in the $\mathrm{MCl}$ group (two eyes in 68 patients, one eye in 2 patients ( 2 right)), 91 in the middle-stage $A D$ group (two eyes in 37 patients, one eye in 17 patients (6 right and 11 left)), and 105 in the control group (two eyes in 51 patients, one eye in 3 patients (3 right)) (Table 1).

No statistically significant difference was found between the middle-stage $A D, M C l$, and control groups in terms of mean, superior, and inferior GCC thickness in the OCT examination (Table 2). The effects of $\mathrm{MCl}$ and middle-stage AD on GCC thicknesses were evaluated by multivariate linear regres- sion analysis by making adjustments according to age and gender. It was determined that the mean, superior and inferior GCC thicknesses of the control group and $\mathrm{MCl}$ and middle-stage $\mathrm{AD}$ groups were statistically similar.

When the control, $\mathrm{MCl}$, and middle-stage $A D$ groups were compared in terms of RNFL thickness, except for the inferior quadrant and the inferior temporal sector, RNFL thicknesses of the other quadrants and sectors were statistically similar (Table 3). It was determined that inferior quadrant RNFL thicknesses showed a statistically significant difference between the groups ( $p=0.007$ ), and inferior RNFL was thinner in the middle-stage AD group compared to the control and $\mathrm{MCl}$ groups ( $p=0.014$ and $p=0.020$, respectively). (Table 3 ).

Table 1. Demographic characteristics, MMT scores of control, MCl and middle-stage AD groups, and the number of patients for whom OCT was evaluated bilaterally or unilaterally

\begin{tabular}{|c|c|c|c|c|}
\hline & $\begin{array}{c}\text { Control } \\
(n=54)\end{array}$ & $\underset{(n=70)}{\mathrm{MCl}}$ & Middle-stage AD ( $n=54)$ & $\mathbf{P}$ \\
\hline Age (years) * & $68.0(9.25)^{a . b}$ & $72.5(10.00)^{a}$ & $77.0(16.00)^{b}$ & $<0.001 \dagger$ \\
\hline Gender ** & & & & $0.021 \neq$ \\
\hline Male & $22(\% 40.7)^{a}$ & $42(\% 60.0)^{\text {a.c }}$ & $20(\% 37.0)^{c}$ & \\
\hline Female & $32(\% 59.3)^{a}$ & $28(\% 40.0)^{a . c}$ & $34(\% 63.0)^{c}$ & \\
\hline $\mathrm{MMT}$ * & $28.0(2.00)^{a . b}$ & $25.0(3.00)^{a . c}$ & $13.0(5.00)^{b . c}$ & $<0,001 \dagger$ \\
\hline \multicolumn{5}{|c|}{ OCT (number of patients, \%) } \\
\hline Bilateral & $51(\% 94.4)$ & 68 (\%97.1) & $37(\% 68.5)$ & \\
\hline Unilateral & $3(\% 5.6)$ & $2(\% 2.9)$ & $17(\% 31.5)$ & \\
\hline Right & $3(\% 5.6)$ & $2(\% 2.9)$ & $6(\% 11.1)$ & \\
\hline Left & $0(\% 0,0)$ & $0(\% 0,0)$ & $11(\% 20,4)$ & \\
\hline
\end{tabular}

* Descriptive statistics shown as median (IQR), ** Data presented as number of cases and (\%), † Kruskal-Wallis test, $\neq$ Pearson's Chi-square test, a: Control vs. $\mathrm{MCl}(\mathrm{p}<0.05), \mathrm{b}$ : Control vs. middle-stage $\mathrm{AD}(\mathrm{p}<0.001), \mathrm{c}$ : $\mathrm{MCl}$ vs. middle-stage $A D(p<0.05)$. MCl: Mild cognitive impairment, AD: Alzheimer's disease, MMT: Mini Mental Test 
Table 2. GCC thicknesses in control, MCl, and mid-stage AD groups

\begin{tabular}{|l|c|c|c|c|}
\hline & Control & MCI & Middle-stage AD & P † \\
\hline Average & $96.75(10.75)$ & $94.00(12.00)$ & $95.75(14.63)$ & 0.097 \\
\hline Superior & $96.75(9.75)$ & $92.75(10.75)$ & $95.00(10.63)$ & 0.065 \\
\hline Inferior & $96.75(12.38)$ & $96.00(11.00)$ & $95.25(14.63)$ & 0.270 \\
\hline
\end{tabular}

* Data shown as median (IQR), † Kruskal-Wallis test. MCl: Mild cognitive impairment, AD: Alzheimer's disease, MMT: Mini Mental Test, The unit for GCC thickness value is $\mu \mathrm{m}$.

Inferior temporal sector RNFL thicknesses showed a statistically significant difference between the groups ( $p=0.002)$; it was observed that inferior temporal RNFL was thinner in the middle-stage $A D$ group compared to the control and $\mathrm{MCl}$ groups ( $p$ $=0.002$ and $p=0.021$, respectively). (Table 3).

When adjusted for age and gender, inferior RNFL ( $\mathrm{B}=-0.054 ; 95 \% \mathrm{Cl}:-0.103--0.005$ and $p=$ 0.030 ) and inferior temporal RNFL were found to be thinner ( $\mathrm{B}=-0.088$; $95 \% \mathrm{Cl}:-0.153$ to -0.023 and $\mathrm{p}=$ 0.008 ) in the middle-stage AD group compared to the control group (Table 4). Again, when the effects of age and gender were kept constant, nasal RNFL ( $\mathrm{B}=0.098 ; 95 \% \mathrm{Cl}: 0.013-0.183$ and $p=0.024$ ) and nasal lower RNFL ( $\mathrm{B}=0.111 ; 95 \% \mathrm{Cl}: 0.020-0.202$ and $p=0.017$ ) were found to be thicker in the middle-stage AD group compared to the control group (Table 4).

Table 3. RNFL thicknesses in control, MCl, and middle-stage AD groups

\begin{tabular}{|c|c|c|c|c|}
\hline & Control & $\mathrm{MCl}$ & Middle-stage AD & $p \dagger$ \\
\hline Average & $97.75(11.25)$ & 97.25 (14.38) & 96.25 (14.63) & 0.664 \\
\hline Superior & $119.75(20.25)$ & $114.25(15.25)$ & $112.50(17.75)$ & 0.215 \\
\hline Temporal & $75.00(11.25)$ & 71.75 (14.13) & 70.50 (19.50) & 0.284 \\
\hline Inferior & $119.50(13.63)^{a}$ & $120.00(19.00)^{b}$ & $111.50(19.50)^{a . b}$ & 0.007 \\
\hline Nasal & $79.25(14.75)$ & $80.00(21.38)$ & $81.00(25.00)$ & 0.136 \\
\hline Superior nasal & $105.75(18.00)$ & 101.75 (21.63) & $102.50(23.00)$ & 0.512 \\
\hline Superior temporal & $125.00(21.75)$ & $125.50(19.13)$ & 119.25 (26.88) & 0.083 \\
\hline Temporal upper & $79.75(12.13)$ & $75.50(13.63)$ & 77.75 (24.38) & 0.291 \\
\hline Temporal lower & $66.00(11.25)$ & $65.75(11.50)$ & $61.50(19.50)$ & 0.253 \\
\hline Inferior temporal & $128.75(18.00)^{a}$ & $126.75(18.88)^{b}$ & $115.00(25.00)^{a . b}$ & 0.002 \\
\hline Inferior nasal & 109.25 (19.88) & $112.00(24.25)$ & $106.25(23.88)$ & 0.377 \\
\hline Nasal lower & $74.75(15.25)$ & $76.25(23.00)$ & $80.00(30.88)$ & 0.100 \\
\hline Nasal upper & 82.75 (17.13) & 81.75 (21.88) & $83.75(29.63)$ & 0.288 \\
\hline
\end{tabular}

* Descriptive statistics shown as median (IQR), † Kruskal-Wallis test, a: Control vs. middle-stage $A D(p<0.05), b: M C l$ vs. middle-stage $A D(p<0.05)$. MCl: Mild cognitive impairment, AD: Alzheimer's disease, MMT: Mini Mental Test, The unit for RNFL thickness value is $\mu m$. 
Table 4. Effects of $\mathrm{MCl}$ and middle-stage AD on RNFL thickness according to the control group when adjusted for age and gender with multivariate linear regression analysis

\begin{tabular}{|c|c|c|c|c|c|}
\hline \multirow[b]{2}{*}{ Average } & \multirow[t]{2}{*}{ B } & \multicolumn{2}{|c|}{$\mathrm{LL} \quad{ }^{95 \% \mathrm{Cl} \text { for } \mathrm{B}} \mathrm{UL}$} & \multirow[t]{2}{*}{$t$} & \multirow[t]{2}{*}{$p+$} \\
\hline & & & & & \\
\hline $\mathrm{MCl}$ & 0.003 & -0.032 & 0.038 & 0.179 & 0.858 \\
\hline Middle-stage AD & 0.001 & -0.037 & 0.039 & 0.056 & 0.956 \\
\hline \multicolumn{6}{|l|}{ Superior } \\
\hline $\mathrm{MCl}$ & -0.007 & $-0,052$ & 0.039 & -0.293 & 0.770 \\
\hline Middle-stage AD & -0.019 & -0.069 & 0.030 & -0.761 & 0.448 \\
\hline \multicolumn{6}{|l|}{ Temporal } \\
\hline $\mathrm{MCl}$ & -0.009 & -0.066 & 0.047 & -0.323 & 0.747 \\
\hline Middle-stage AD & -0.021 & -0.083 & 0.041 & -0.678 & 0.499 \\
\hline \multicolumn{6}{|l|}{ Inferior } \\
\hline $\mathrm{MCl}$ & 0.001 & -0.043 & 0.046 & 0.053 & 0.958 \\
\hline Middle-stage AD & -0.054 & -0.103 & -0.005 & -2.186 & 0.030 \\
\hline \multicolumn{6}{|l|}{ Nasal } \\
\hline $\mathrm{MCl}$ & 0.046 & -0.032 & 0.123 & 1.156 & 0.249 \\
\hline Middle -stage AD & 0.098 & 0.013 & 0.183 & 2.274 & 0.024 \\
\hline \multicolumn{6}{|l|}{ Superior nasal } \\
\hline $\mathrm{MCl}$ & -0.028 & -0.085 & 0.030 & -0.953 & 0.342 \\
\hline Middle -stage AD & -0.016 & -0.078 & 0.046 & -0.498 & 0.619 \\
\hline \multicolumn{6}{|l|}{ Superior temporal } \\
\hline $\mathrm{MCl}$ & 0.015 & -0.046 & 0.077 & 0.491 & 0.624 \\
\hline Middle -stage AD & -0.014 & -0.080 & 0.053 & -0.401 & 0.689 \\
\hline \multicolumn{6}{|l|}{ Temporal upper } \\
\hline $\mathrm{MCl}$ & -0.028 & -0.090 & 0.034 & -0.898 & 0.370 \\
\hline Middle -stage AD & -0.003 & -0.070 & 0.063 & -0.097 & 0.923 \\
\hline \multicolumn{6}{|l|}{ Temporal lower } \\
\hline $\mathrm{MCl}$ & 0.007 & -0.065 & 0.079 & 0.196 & 0.844 \\
\hline Middle -stage AD & -0.037 & -0.116 & 0.041 & -0.941 & 0.348 \\
\hline \multicolumn{6}{|l|}{ Inferior temporal } \\
\hline $\mathrm{MCl}$ & -0.004 & -0.065 & 0.056 & -0.144 & 0.886 \\
\hline Middle -stage AD & -0.088 & -0.153 & -0.023 & -2.672 & 0.008 \\
\hline \multicolumn{6}{|l|}{ Inferior nasal } \\
\hline $\mathrm{MCl}$ & 0.010 & -0.051 & 0.071 & 0.316 & 0.752 \\
\hline Middle -stage AD & -0.013 & -0.079 & 0.053 & -0.395 & 0.693 \\
\hline \multicolumn{6}{|l|}{ Nasal lower } \\
\hline $\mathrm{MCl}$ & 0.043 & -0.041 & 0.127 & 1.012 & 0.313 \\
\hline Middle -stage AD & 0.111 & 0.020 & 0.202 & 2.406 & 0.017 \\
\hline \multicolumn{6}{|l|}{ Nasal upper } \\
\hline $\mathrm{MCl}$ & 0.019 & -0.060 & 0.098 & 0.472 & 0.637 \\
\hline Middle -stage AD & 0.077 & -0.009 & 0.162 & 1.776 & 0.077 \\
\hline
\end{tabular}

Cl: Confidence interval, LL: Lower limit, UL: Upper limit. MCl: Mild cognitive impairment, AD: Alzheimer's disease 
When adjusted for age and gender, inferior RNFL ( $\mathrm{B}=-0.055 ; 95 \% \mathrm{Cl}:-0.100--0.010$ and $p=$ $0.016)$ and inferior temporal RNFL ( $B=-0.084 ; 95 \%$ $\mathrm{Cl}$ : $-0.144--0.024$ and $p=0.007)$ were thinner in the middle-stage $A D$ group compared to the $\mathrm{MCl}$ group (Table 5).

\section{DISCUSSION}

Our study results indicated that RNFL was affected in middle-stage $A D$ patients and that this involvement was limited to inferior and inferior temporal RNFL regions. Despite the effect on RNFL, no change in GCC thickness was found in patients with middle-stage $A D$ and $\mathrm{MCl}$. Data obtained from histopathological studies on retinal involvement in $A D$ showed a significant loss of retinal ganglion cells and a decrease in RNFL thickness in individuals with AD compared to the controls $(5,10,12)$.

It has been suggested that it may be possible to affect specific retinal cell types in $A D$; in particular, the ganglion cell-inner plexiform layer (GC-IPL) complex in the macula has been associated with $A D$ (13). It has also been suggested that the measurement of macular GC-IPL thickness would be a suitable predictor for $A D$ (14). On the other hand, if ganglion cells are damaged throughout the retina, this can be best evaluated by peripapillary RNFL measurements that reflect all axonal bumps that leave the retina to join the optic nerve. The thickness of RNFL has also been thought to decrease in AD due to retrograde degeneration of retinal ganglion cell axons (6).

In our study, although there was thinning in RNFL in $A D$ patients, no change was detected in GCC thickness, and it was thought that this finding could be explained by the metabolic failure and energy deprivation caused by neurodegenerative events, primarily by affecting the axons of ganglion cells. In a population-based prospective cohort study, thinner RNFL thickness was associated with the risk of developing dementia, including $A D$, independent of cardiovascular risk factors (10). Our study showed selectivity in the decrease in RNFL thickness in middle-stage AD patients; it was determined that the inferior and, especially, the inferior temporal region were affected. We thought that RNFL measurements in these regions could better reflect neurodegeneration and axon loss associated with AD.

Our study found that, after multivariate linear regression analysis, RNFL thickening was found in the nasal and nasal lower regions in the AD group compared to the control group. However, the clinical significance of this finding is not fully understood. While most OCT studies have reported a general thinning in RNFL by combining data from all quadrants (15-17), other studies have reported that RNFL thinning is more pronounced and selective in the inferior (18-20) or superior quadrants (21). It was found that inferior quadrant RNFL thickness had the strongest correlation with the results of cognitive tests, and the risk of cognitive decline was higher in older adults with RNFL thinning in this quadrant (18).

The answer to the question of why the degenerative process affects certain retinal regions in $A D$ is unclear. It has been suggested that the superior and inferior quadrants contain more large-diameter axons, which degenerate more rapidly, and which may be the reason for the sensitivity of these quadrants (22). Another possibility that comes to mind regarding regional involvement in the retina is that the pathology in the primary brain regions affected by the neurodegenerative process associated with $A D$ shows somatotopic organization while reflecting on retinal nerve fibers, which can be proven by advanced histopathological and imaging studies.

As our study results indicate, focal damage can occur in RNFL in AD. Therefore, it may be a useful approach to investigate the potential focal effects of $A D$ and measure changes in the most affected area of the retina using customized nerve fiber layer thickness maps or other new techniques. However, with the disease's progression, retinal nerve fibers 
Table 5. Effects of control and middle-stage AD on RNFL thickness according to the $\mathrm{MCl}$ group when adjusted for age and gender with multivariate linear regression analyses

\begin{tabular}{|c|c|c|c|c|c|}
\hline \multirow[b]{2}{*}{ Average } & \multirow[t]{2}{*}{ B } & \multicolumn{2}{|c|}{${ }_{\mathrm{LL}}^{95 \% \mathrm{Cl} \text { for } \mathrm{B}} \mathrm{UL}$} & \multirow[t]{2}{*}{$\mathbf{t}$} & \multirow[t]{2}{*}{$p+$} \\
\hline & & & & & \\
\hline Control & -0.003 & -0.038 & 0.032 & -0.179 & 0.858 \\
\hline Middle-stage AD & -0.002 & -0.037 & 0.033 & -0.119 & 0.906 \\
\hline \multicolumn{6}{|l|}{ Superior } \\
\hline Control & 0.007 & -0.039 & 0.052 & 0.293 & 0.770 \\
\hline Middle-stage AD & -0.012 & -0.058 & 0.033 & -0.534 & 0.594 \\
\hline \multicolumn{6}{|l|}{ Temporal } \\
\hline Control & 0.009 & -0.047 & 0.066 & 0.323 & 0.747 \\
\hline Middle-stage AD & -0.012 & -0.069 & 0.045 & -0.414 & 0.679 \\
\hline \multicolumn{6}{|l|}{ Inferior } \\
\hline Control & -0.001 & -0.046 & 0.043 & -0.053 & 0.958 \\
\hline Middle-stage AD & -0.055 & -0.100 & -0.010 & -2.425 & 0.016 \\
\hline \multicolumn{6}{|l|}{ Nasal } \\
\hline Control & -0.046 & -0.123 & 0.032 & -1.156 & 0.249 \\
\hline Middle-stage AD & 0.052 & -0.026 & 0.131 & 1.319 & 0.189 \\
\hline \multicolumn{6}{|l|}{ Superior Nasal } \\
\hline Control & 0.028 & -0.030 & 0.085 & 0.953 & 0.342 \\
\hline Middle-stage AD & 0.012 & -0.045 & 0.069 & 0.414 & 0.679 \\
\hline \multicolumn{6}{|l|}{ Superior temporal } \\
\hline Control & -0.015 & -0.077 & 0.046 & -0.491 & 0.624 \\
\hline Middle-stage AD & -0.029 & -0.091 & 0.033 & -0.924 & 0.357 \\
\hline \multicolumn{6}{|l|}{ Temporal upper } \\
\hline Control & 0.028 & -0.034 & 0.090 & 0.898 & 0.370 \\
\hline Middle-stage AD & 0.025 & -0.037 & 0.086 & 0.793 & 0.429 \\
\hline \multicolumn{6}{|l|}{ Temporal lower } \\
\hline Control & -0.007 & -0.079 & 0.065 & -0.196 & 0.844 \\
\hline Middle-stage AD & -0.045 & -0.117 & 0.028 & -1.215 & 0.226 \\
\hline \multicolumn{6}{|l|}{ Inferior temporal } \\
\hline Control & 0.004 & -0.056 & 0.065 & 0.144 & 0.886 \\
\hline Middle-stage AD & -0.084 & -0.144 & -0.024 & -2.749 & 0.007 \\
\hline \multicolumn{6}{|l|}{ Inferior Nasal } \\
\hline Control & -0.010 & -0.071 & 0.051 & -0.316 & 0.752 \\
\hline Middle-stage AD & -0.023 & -0.084 & 0.038 & -0.744 & 0.458 \\
\hline \multicolumn{6}{|l|}{ Nasal lower } \\
\hline Control & -0.043 & -0.127 & 0.041 & -1.012 & 0.313 \\
\hline Middle-stage AD & 0.068 & -0.016 & 0.152 & 1.593 & 0.113 \\
\hline \multicolumn{6}{|l|}{ Nasal upper } \\
\hline Control & -0.019 & -0.098 & 0.060 & -0.472 & 0.637 \\
\hline Middle-stage AD & 0.058 & -0.021 & 0.137 & 1.451 & 0.149 \\
\hline
\end{tabular}

Cl: Confidence interval, LL: Lower limit, UL: Upper limit. MCl: Mild cognitive impairment, AD: Alzheimer's disease 
may be affected in all regions, and to demonstrate this, evaluations or long-term follow-up studies are required in advanced-stage $A D$.

Our study found that RNFL was affected only in patients with middle-stage $A D$; similar effects were not observed in patients with $\mathrm{MCl}$, and no difference was found between patients with $\mathrm{MCl}$ and healthy individuals in terms of retinal involvement. It was thought that retinal degeneration findings might run parallel to the progression of $A D$ pathology, and therefore, while OCT was affected in AD patients, normal findings could be detected in $\mathrm{MCl}$. Although the number of studies evaluating OCT findings in $\mathrm{MCl}$ patients is relatively small, several studies have reported OCT abnormalities in patients with $\mathrm{MCl}(17,19,23)$. Attention was drawn to the relationship between inferior quadrant RNFL thickness and episodic memory in $\mathrm{MCl}$ patients; it has been suggested that it can be used as a biomarker in $\mathrm{MCl}$ and $\mathrm{AD}$ patients (20).

While our study found no abnormality in OCT in the control group and $\mathrm{MCl}$ patients, changes in RNFL thickness were detected only in mid-stage $A D$, and it was thought that $O C T$ findings might not have a predictive feature for $\mathrm{MCl}$ or $\mathrm{AD}$. The definition of $\mathrm{MCl}$ shows a heterogeneous feature since it includes cognitive disorders that can turn into different types of dementia. While patients diagnosed with $\mathrm{MCl}$ were not sub-grouped in most other OCT studies, in our study, patients with amnestic $\mathrm{MCl}$ with a high probability of conversion to $A D$ were selected; non-amnestic $\mathrm{MCl}$, which may be the pre-

\section{REFERENCES}

1. Ong Y-L, Ong Y-T, Ikram MK, Chen CLH, Wong TY. Potential Applications of Spectral-Domain Optical Coherence Tomography (SD-OCT) in the Study of Alzheimer's Disease. Proceedings of Singapore Healthcare. 2014;23(1):74-83. (DOI:10.1177/2010105 81402300112).

2. Moschos MM, Markopoulos I, Chatziralli l et al. Structural and functional impairment of the retina and op- stage of other dementias, were excluded. We considered this selectivity preferable to the ones used in other studies.

The absence of early or advanced AD patients in our study groups may have restricted the OCT examination to give clearer results concerning the stages of the disease; However, it may be challenging to differentiate mild stage $A D$ from $\mathrm{MCl}^{\text {; It may }}$ not be possible for patients with advanced-stage $A D$ to adapt to OCT and other eye examinations. Another limitation of our study was the statistical difference between the groups in terms of age and gender distribution. However, the possible effect of this difference in other analyzes was eliminated by making statistical corrections according to age and gender. Due to the difficulty of cooperation and ocular reasons specified in the exclusion criteria, OCT examination could be performed in only one eye in some of the patients in the patient groups, and the data belonging to one eye were evaluated. This was a limitation of our study due to the participants' advanced age or impaired cognitive functions, or both.

In conclusion, our study results indicated that retinal axon loss might develop in $A D$, that this loss may have a focal feature, and that the inferior temporal region may have a selective sensitivity. OCT can reflect the neuropathological process in $A D$ and could contribute to patients' diagnosis and follow-up. However, to fully enlighten the predictive value of OCT, long-term studies covering all stages of the disease will be needed.

tic nerve in Alzheimer's disease. Curr Alzheimer Res 2012;9: 782-788. (PMID: 22698074).

3. Lee MJ, Abraham AG, Swenor BK et al. Application of optical coherence tomography in the detection and classification of cognitive decline. J Curr Glaucoma Pract 2018 Jan-Apr;12(1):10-18. (PMID: 29861577).

4. Grimaldi A, Brighi C, Peruzzi G et al. Inflammation, neurodegeneration and protein aggregation in the 
retina as ocular biomarkers for Alzheimer's disease in the 3xTg-AD mouse model. Cell Death Dis 2018 Jun 7;9(6): 685. (PMID: 29880901).

5. Hinton DR, Sadun AA, Blanks JC et al. Optic-nerve degeneration in Alzheimer's disease. N Engl J Med 1986 Aug 21;315(8): 485-7. (PMID: 3736630).

6. Ascaso FJ, Cruz N, Modrego PJ et al. Retinal alterations in mild cognitive impairment and Alzheimer's disease: an optical coherence tomography study. J Neurol 2014 Aug;261(8): 1522-30. (PMID: 24846203).

7. Garcia-Martin ES, Rojas B, Ramirez A et al. Macular thickness as a potential biomarker of mild Alzheimer's disease. Ophthalmology 2014 May;121(5): 1149 1151.e3. (PMID: 24656417).

8. Thomson KL, Yeo JM, Waddell B, Cameron JR, Pal S. A systematic review and meta-analysis of retinal nerve fiber layer change in dementia, using optical coherence tomography. Alzheimers Dement (Amst) 2015 Apr 23;1(2): 136-43. (PMID: 27239501).

9. Ko F, Muthy ZA, Gallacher J et al. Association of retinal nerve fiber layer thinning with current and future cognitive decline: a study using optical coherence tomography. JAMA Neurol 2018 Jun 25. (PMID: 29946685).

10. Mutlu U, Colijn JM, Ikram MA et al. Association of retinal neurodegeneration on optical coherence tomography with dementia: a population-based study. JAMA Neurol 2018 Jun 25. (PMID: 29946702).

11. Cruz-Herranz A, Balk LJ, Oberwahrenbrock $T$ et al. The APOSTEL recommendations for reporting quantitative optical coherence tomography studies. Neurology 2016 Jun 14;86(24): 2303-9. (PMID: 27225223).

12. Blanks JC, Hinton DR, Sadun AA, Miller CA. Retinal ganglion cell degeneration in Alzheimer's disease. Brain Res 1989;501(2): 364-372. (PMID: 2819446).

13. Cheung $C Y$, Ong $Y T$, Hilal $S$ et al. Retinal ganglion cell analysis using high-definition optical coherence tomography in patients with mild cognitive impairment and Alzheimer's disease. J Alzheimers Dis 2015;45(1): 45-56. (PMID: 25428254).

14. Mwanza J-C, Oakley JD, Budenz DL et al. Macular ganglion cell-inner plexiform layer: automated detection and thickness reproducibility with spectral domain-optical coherence tomography in glauco- ma. Invest Ophthalmol Vis Sci 2011 Oct;52(11): 83238329. (PMID: 21917932).

15. Marziani E, Pomati S, Ramolfo P, et al. Evaluation of retinal nerve fiber layer and ganglion cell layer thickness in Alzheimer's disease using spectral-domain optical coherence tomography. Invest Ophthalmol Vis Sci 2013;54(9): 5953-5958. (PMID: 23920375).

16. Iseri PK, Altinaş $O$, Tokay $T$, Yüksel N. Relationship between cognitive impairment and retinal morphological and visual functional abnormalities in Alzheimer disease. J Neuroophthalmol. 2006 Mar;26(1):18-24. (PMID: 16518161).

17. He XF, Liu YT, Peng C, et al. Optical coherence tomography assessed retinal nerve fiber layer thickness in patients with Alzheimer's disease: a metaanalysis. Int J Ophthalmol 2012 Jun;5(3): 401-405. (PMID: 22773997).

18. Shi $Z$, Wu $Y$, Wang $M$ et al. Greater attenuation of retinal nerve fiber layer thickness in Alzheimer's disease patients. J Alzheimers Dis 2014;40(2): 277-283. (PMID: 24413621).

19. Kesler A, Vakhapova V, Korczyn AD, Naftaliev E, Neudorfer M. Retinal thickness in patients with mild cognitive impairment and Alzheimer's disease. Clin Neurol Neurosurg 2011 Sep;113(7): 523-526. ( PMID: 21454010).

20. Shen Y, Liu L, Cheng $Y$ et al. Retinal nerve fiber layer thickness is associated with episodic memory deficit in mild cognitive impairment patients. Curr Alzheimer Res 2014 Mar;11(3): 259-266. (PMID: 24484274).

21. Kirbas S, Turkyilmaz K, Anlar O, Tufekci A, Durmus M. Retinal nerve fiber layer thickness in patients with Alzheimer disease. J Neuroophthalmol 2013 Mar;33(1): 58-61. (PMID: 22918296)

22. Cabrera DeBuc D, Gaca-Wysocka M, Grzybowski A, Kanclerz P. Identification of Retinal Biomarkers in Alzheimer's Disease Using Optical Coherence Tomography: Recent Insights, Challenges, and Opportunities. J Clin Med. 2019 Jul 9;8(7):996. (PMID: 31323964).

23. Liu D, Zhang L, Li Z et al. Thinner changes of the retinal nerve fiber layer in patients with mild cognitive impairment and Alzheimer's disease. BMC Neurol 2015 Feb;15: 14. (PMID: 25886372). 